\title{
Profile of rural tourism consumers in Serbia and Austria'
}

\section{Профил српских и аустријских потрошача руралног туризма}

\author{
Slavica Tomić \\ University of Novi Sad, Faculty of Economics in Subotica, Republic of Serbia, slavica.tomic@ef.uns.ac.rs \\ Ksenija Leković \\ University of Novi Sad, Faculty of Economics in Subotica, Republic of Serbia, ksenija.lekovic@ef.uns.ac.rs \\ Pernille Eskerod \\ Webster Vienna Private University, Faculty - Business and Management, Republic of Austria, \\ pernille.eskerod@webster.ac.at \\ Eva Zedlacher \\ Webster Vienna Private University, Faculty - Business and Management, Republic of Austria, \\ eva.zedlacher@webster.ac.at
}

\begin{abstract}
In order to achieve successful positioning, rural tourism managers and marketers need to understand consumer behaviour. Above all, they need to answer the question of which customer profiles are suitable to particular tourist destinations There are three types of statistical measurement used for research into consumer, i.e. tourist behaviour, and those are volume, value and profile. A profile encompasses characteristics of the consumers per se and characteristics of trae. The aim of the paper is to define the profile of rural tourism consumer based on the results of the conducted research, taking into account two socio-demographic characteristics of the consumers - age and education level in relation to three characteristics of travel - frequency of engaging in rural tourism, travel companions and information sources used to plan the trip. The research encompassed 150 respondents each from Serbia and Austria respectively. Cross-tabulation method was used for testing the set hypotheses
\end{abstract}

Keywords: rural tourism, consumers of rural tourism, profile, homestead, wine tourism JEL classification: M21, M30, Z30

Сажетак: У циљу успешног позиционирања на менаџерима и маркетарима руралног туризма је да се упознају са понашањем потрошача. Првенствено морају да одговоре на питање који профил потрошача одговара одређеној туристичкој дестинацији? Три су статистичка мерила која се користе како би се истражило понашање потрошача (туриста) и то су: обим, вредност и профил. Профил обједињује карактеристике самог потрошача и карактеристике путовања. Циљ рада је да се на основу резултата спроведеног истраживања дефинише профил потрошача руралног туризма узимајући у обзир две социодемографске карактеристике потрошача, узраст и ниво образовања, у поређењу са три карактеристике путовања: учесталост посете руралној туристичкој дестинацији, пратиоци на путовању и извори информација које потрошачи користе приликом планирања посете руралној туристичкој

${ }^{1}$ This paper is a part of the bilateral project „Stakeholder engagement within rural tourism in Austria and Serbia“, which is under the auspices of the Ministry of Education, Science and Technological Development of the Republic of Serbia, project number: 451-0302141/2017-09/09

Corresponding author 
дестинацији. Истраживањем су обухваћена два облика руралног туризма, салаши и вински туризам. Узорак је обухватио по 150 испитаника са територије Републике Србије и Републике Аустрије. У циљу тестирања постављених хипотеза примењен је метод унакрсног табелирања.

Кључне речи: рурални туризам, потрошачи руралног туризма, профил, салаш, вински туризам JEЛ класификација: M21, M30, Z30

\section{Introduction}

As a separate branch of tourism, rural tourism can be defined as hospitality providing rural environment for consumers (Erdeji et al., 2013). Despite the fact that consumers, i.e. tourists, experience the "quaint" lifestyle during their visits to tourist destinations, a need imposes itself to adapt the offer to their needs and expectations (Grubor et al., 2019) as a rule, the offer of rural tourism encompasses festivals, events, as well as manufacture and sale of handicrafts and agricultural products. It also includes numerous additional activities arranged by rural hosts such as hunting, fishing, horse-riding, walking, even wellness (Đorđević et al., 2019; Quendler, 2020).

A more intense development of rural tourism in Europe started in the second half of the $20^{\text {th }}$ century, as a results of consumers' enormous wish to relate to nature, discover new landscapes, new cultures and customs. Nowadays, it accounts for a noteworthy portion of the global tourist offer. An estimation of the share of consumers of rural tourism in the total number of tourists is 3\% on the global level and 25\% within the EU (Radović \& Vasiljević, 2018). Rural tourism in Serbia takes up almost $25 \%$ of all forms of tourist activities (Stepanov et al., 2018) whereas rural tourism in Austria accounts for more than $80 \%$ of Austrian national tourism (Breiling, 2005).

Rural tourism managers and marketers should take into account what kind of consumers they want to attract for a particular rural tourist destination. In other words, they need to answer the question, what is the profile of those consumers? Consumers of rural tourism in Serbia and Austria are the subject of this paper. The aim was to define the profile of the rural tourism consumer based on the results of the conducted research, taking into account two socio-demographic characteristics of the consumer - age and education level in relation to three characteristics of travel - frequency of engaging in rural tourism, travel companions and information sources used to plan the trip. In this, the research was limited to two forms of rural tourism - homesteads and wineries.

\section{Theoretical background}

In order to achieve successful positioning rural tourism managers and marketers need to understand consumer behaviour. Necessary information can be obtained by researching habits, desires and needs of consumers of rural tourism.

Cooper \& Schindler (2006) propose three types of statistical measurement used for research into consumer behaviour of tourists, and those are volume, value and profile. Volume includes elements such as the number of overnight stays and average duration of visit. Value includes all elements pertaining to the budget of the intended travel, such as the 
amount of money spent per day, the amount allocated for lodging, dining etc. Profile, as statistical measurement, incorporates the characteristics of the consumers themselves and the characteristic and the characteristics of the travel.

Socio-demographic factors frequently used in the process of forecasting tourists' behaviour patterns are: age, gender, family life cycle, education and income (Colins \& Tisdell, 2002; Foot, 2004; Ma et al., 2018; Kara \& Mkwizu, 2020). According to Koteski et al. (2017) there are multiple types, i.e. profiles of consumers of rural tourism: day-trippers, visitors on a short holiday, families, elderly visitors, special interest tourists and educational groups. Further, Đenadić et al. (2016) deduce that the majority of the consumers of rural tourism are families with children and couples, and the second largest are groups of friends and young people.

On the other hand, there are several types, i.e. forms of rural tourism. What is significant from the aspect of this paper are homesteads and wineries. Farm, i.e. homestead tourism is characteristic of Vojvodina and features as a well-developed and popular rural tourism product (Radović \& Vasiljević, 2018). Homesteads used to be isolated, plain farms. Today, some of them are Skansen-like museums with a diversity of heirloom objects, household furnishings and farming tools. Farms and homesteads have retained the traditional lifestyle while adapting it to the modern way of life, so that many of them have swimming pools, sports facilities, fish ponds and miniature ZOOs. (Medojević et al., 2011). From the cultural and tourist point of view, homesteads are distinctive marks of the Province of Vojvodina. (Vujko et al., 2017). Wine tourism refers to travelling down wine routes (Radović \& Vasiljević, 2018) and includes vine cellars, wine tasting, culinary experience, leisure and cultural activities (Vasiljević \& Vujović, 2012). The main motivation of this branch of rural tourism is to sample local cuisine and wine (Hall et al., 2002; Erdeji et al., 2013).

\section{Methodology}

The aim of the paper is to point to the existence of differences in consumer/tourists' age and education levels in relation to frequency of engaging in rural tourism, travel companions and sources of information the consumers use when planning and making decisions on visiting particular tourist destinations. The paper presents a part of the results of research conducted on convenience samples of 150 respondents each od different and education levels from the territories of Serbia and Austria. The only condition for participating in the research was that the respondents had visited a rural tourist destination in the past two years. In Serbia, this condition was limited to homesteads and wineries in Vojvodina, or wineries around Vienna. The research was conducted in April and May, by sending an online questionnaire consisting of three parts, drafted and adapted from the questionnaire used by Polo Pena et al. (2012) and Alcaniz et al. (2009) in their research studies. Results presented in this paper were obtained based on data from the first two parts of the questionnaires regarding respondents' socio-demographic characteristics and characteristics of travel. The presentation of characteristics of respondents included in this research is given in Table 1. 
Table 1: Profile of survey respondents

\begin{tabular}{|c|c|c|c|c|c|}
\hline \multirow{2}{*}{\multicolumn{2}{|c|}{ Variables }} & \multicolumn{2}{|c|}{ Serbia } & \multicolumn{2}{|c|}{ Austria } \\
\hline & & \multirow{2}{*}{$\begin{array}{c}\begin{array}{c}\text { Frequency } \\
(\mathrm{n}=150)\end{array} \\
40\end{array}$} & \multirow{2}{*}{$\begin{array}{c}\text { Percentage } \\
26.7 \\
\end{array}$} & \multirow{2}{*}{$\begin{array}{c}\begin{array}{c}\text { Frequency } \\
(\mathrm{n}=150)\end{array} \\
53 \\
\end{array}$} & \multirow{2}{*}{$\frac{\text { Percentage }}{35.3}$} \\
\hline \multirow{5}{*}{ Age } & $18-29$ & & & & \\
\hline & $30-39$ & 56 & 37.3 & 41 & 27.3 \\
\hline & $40-49$ & 29 & 19.3 & 33 & 22.0 \\
\hline & $50-59$ & 18 & 12.0 & 16 & 10.7 \\
\hline & $\geq 60$ & 7 & 4.7 & 7 & 4.7 \\
\hline \multirow{5}{*}{ Education level } & Primary school & 1 & 0.7 & 1 & 0.7 \\
\hline & Secondary school & 22 & 14.7 & 25 & 16.7 \\
\hline & Bachelor & 68 & 45.3 & 74 & 49.3 \\
\hline & Master & 37 & 24.7 & 27 & 18.0 \\
\hline & Doctoral & 22 & 14.7 & 23 & 15.3 \\
\hline \multirow{4}{*}{$\begin{array}{l}\text { Frequency of } \\
\text { engaging in rural } \\
\text { tourism }\end{array}$} & Once every two or three years & 35 & 23.3 & 24 & 16.0 \\
\hline & Once or twice a year & 54 & 36.0 & 63 & 42.0 \\
\hline & Three or four times a year & 34 & 22.7 & 19 & 12.7 \\
\hline & More than four times a year & 27 & 18.0 & 44 & 29.3 \\
\hline \multirow{5}{*}{ Travel companions } & Alone & 2 & 1.3 & 1 & 0.7 \\
\hline & Partner & 31 & 20.7 & 29 & 19.3 \\
\hline & Family & 76 & 50.7 & 90 & 60.0 \\
\hline & Friends & 38 & 25.3 & 29 & 19.3 \\
\hline & Other & 3 & 2.0 & 1 & 0.7 \\
\hline \multirow{6}{*}{$\begin{array}{l}\text { Information } \\
\text { sources used to } \\
\text { plan the trip }\end{array}$} & Internet & 74 & 49.3 & 78 & 52.0 \\
\hline & $\begin{array}{lll}\begin{array}{l}\text { Recommendations } \\
\text { and/or relatives }\end{array} & \text { of friends } \\
\end{array}$ & 56 & 37.3 & 49 & 32.7 \\
\hline & Own experience & 20 & 13.3 & 17 & 11.3 \\
\hline & Newspapers/magazines/catalogues & - & - & 1 & 0.7 \\
\hline & Television & - & - & - & - \\
\hline & Travel agencies & - & - & 5 & 3.3 \\
\hline
\end{tabular}

Source: the authors' calculation

The majority of respondents in Serbia were aged between 30 and 39 (37.3\%) with a bachelor's degree (45.3\%), while the majority of respondents in Austria were aged between 18 and $29(35.3 \%)$ with a Bachelor's degree (49.3\%). The majority of the respondents in Serbia (36.0\%) and Austria (42.0\%) visit rural tourist destinations once or twice a year. Respondents in both countries travel most often with their families $(50.7 \%$ in Serbia, $60.0 \%$ in Austria), and Internet is the most frequently used information source when planning a trip (49.3\% in Serbia, 52.0\% in Austria).

Based on the analysed theoretical background and set research objective, the following hypotheses were proposed:

H1: Younger respondents visit rural tourist destinations and use the Internet for planning visits to rural tourist destinations more often, whereas all age groups visit rural tourist destinations with families most often.

H2: Respondents with Bachelor's degrees visit rural tourist destinations more often, and together with respondents with completed secondary school and Master's degrees use the Internet for planning visits to rural tourist destinations, while all education levels of respondents visit rural tourist destinations with families most often 
The hypotheses were tested by means of cross-tabulation method. The statistical software used for data processing and testing the proposed hypotheses is IBM SPSS version 21.

\section{Results and discussion}

Table 2 presents the differences in frequency of visiting tourist destinations according to the respondents' age.

Table 2: Testing the independence of variables: Frequency of Visiting Tourist Destinations and Respondents' Age

\begin{tabular}{|c|c|c|c|c|c|c|c|c|c|}
\hline \multirow{2}{*}{\multicolumn{4}{|c|}{ Country }} & \multicolumn{5}{|c|}{ Age } & \multirow{3}{*}{$\begin{array}{c}\text { Total } \\
35\end{array}$} \\
\hline & & & & \multirow{2}{*}{$\frac{18-29}{8}$} & \multirow{2}{*}{$\frac{30-39}{12}$} & \multirow{2}{*}{$\frac{40-49}{6}$} & \multirow{2}{*}{$\frac{50-59}{5}$} & \multirow{2}{*}{$\frac{\geq 60}{4}$} & \\
\hline \multirow{12}{*}{$\frac{\pi}{0}$} & \multirow{12}{*}{$\begin{array}{l}\text { Frequency of } \\
\text { engaging in } \\
\text { rural tourism }\end{array}$} & \multirow{3}{*}{$\begin{array}{l}\text { Once every two } \\
\text { or three years }\end{array}$} & Count & & & & & & \\
\hline & & & $\%$ Frequency & $22.9 \%$ & $34.3 \%$ & $17.1 \%$ & $14.3 \%$ & $11.4 \%$ & $100.0 \%$ \\
\hline & & & $\%$ Age & $20.0 \%$ & $21.4 \%$ & $20.7 \%$ & $27.8 \%$ & $57.1 \%$ & $23.3 \%$ \\
\hline & & \multirow{3}{*}{$\begin{array}{l}\text { Once or twice a } \\
\text { year }\end{array}$} & Count & 14 & 21 & 12 & 6 & 1 & 54 \\
\hline & & & $\%$ Frequency & $25.9 \%$ & $38.9 \%$ & $22.2 \%$ & $11.1 \%$ & $1.9 \%$ & $100.0 \%$ \\
\hline & & & $\%$ Age & $35.0 \%$ & $37.5 \%$ & $41.4 \%$ & $33.3 \%$ & $14.3 \%$ & $36.0 \%$ \\
\hline & & \multirow{3}{*}{$\begin{array}{l}\text { Three or four } \\
\text { times a year }\end{array}$} & Count & 11 & 10 & 8 & 4 & 1 & 34 \\
\hline & & & $\%$ Frequency & $32.4 \%$ & $29.4 \%$ & $23.5 \%$ & $11.8 \%$ & $2.9 \%$ & $100.0 \%$ \\
\hline & & & $\%$ Age & $27.5 \%$ & $17.9 \%$ & $27.6 \%$ & $22.2 \%$ & $14.3 \%$ & $22.7 \%$ \\
\hline & & \multirow{3}{*}{$\begin{array}{l}\text { More than four } \\
\text { times a year }\end{array}$} & Count & 7 & 13 & 3 & 3 & 1 & 27 \\
\hline & & & $\%$ Frequency & $25.9 \%$ & $48.1 \%$ & $11.1 \%$ & $11.1 \%$ & $3.7 \%$ & $100.0 \%$ \\
\hline & & & $\%$ Age & $17.5 \%$ & $23.2 \%$ & $10.3 \%$ & $16.7 \%$ & $14.3 \%$ & $18.0 \%$ \\
\hline \multirow{12}{*}{ 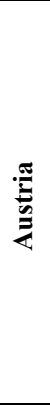 } & \multirow{12}{*}{$\begin{array}{l}\text { Frequency of } \\
\text { engaging in } \\
\text { rural tourism }\end{array}$} & \multirow{3}{*}{$\begin{array}{l}\text { Once every two } \\
\text { or three years }\end{array}$} & Count & 5 & 7 & 8 & 2 & 2 & 24 \\
\hline & & & $\%$ Frequency & $20.8 \%$ & $29.2 \%$ & $33.3 \%$ & $8.3 \%$ & $8.3 \%$ & $100.0 \%$ \\
\hline & & & $\%$ Age & $9.4 \%$ & $17.1 \%$ & $24.2 \%$ & $12.5 \%$ & $28.6 \%$ & $16.0 \%$ \\
\hline & & \multirow{3}{*}{$\begin{array}{l}\text { Once or twice a } \\
\text { year }\end{array}$} & Count & 26 & 15 & 13 & 6 & 3 & 63 \\
\hline & & & $\%$ Frequency & $41.3 \%$ & $23.8 \%$ & $20.6 \%$ & $9.5 \%$ & $4.8 \%$ & $100.0 \%$ \\
\hline & & & $\%$ Age & $49.1 \%$ & $36.6 \%$ & $39.4 \%$ & $37.5 \%$ & $42.9 \%$ & $42.0 \%$ \\
\hline & & \multirow{3}{*}{$\begin{array}{l}\text { Three or four } \\
\text { times a year }\end{array}$} & Count & 3 & 9 & 3 & 3 & 1 & 19 \\
\hline & & & $\%$ Frequency & $15.8 \%$ & $47.4 \%$ & $15.8 \%$ & $15.8 \%$ & $5.3 \%$ & $100.0 \%$ \\
\hline & & & $\%$ Age & $5.7 \%$ & $22.0 \%$ & $9.1 \%$ & $18.8 \%$ & $14.3 \%$ & $12.7 \%$ \\
\hline & & \multirow{3}{*}{$\begin{array}{l}\text { More than four } \\
\text { times a year }\end{array}$} & Count & 19 & 10 & 9 & 5 & 1 & 44 \\
\hline & & & $\%$ Frequency & $43.2 \%$ & $22.7 \%$ & $20.5 \%$ & $11.4 \%$ & $2.3 \%$ & $100.0 \%$ \\
\hline & & & $\%$ Age & $35.8 \%$ & $24.4 \%$ & $27.3 \%$ & $31.3 \%$ & $14.3 \%$ & $29.3 \%$ \\
\hline
\end{tabular}

Source: the authors' calculation

Based on the cross-tabulation of categorical variables Frequency Of Engaging In Rural Tourism, (i.e. Frequency of Visiting Tourist Destinations) and Respondents' Age in Serbia, the most respondents aged 30 to 39 (34.3\%) go once in two or three years, the most respondents aged 30 to $39(38,9 \%)$ go once or twice a year, the most respondents aged 18 to $29(32,4 \%)$ go three or four times a year, and the most respondents aged 30-39 $(48,1)$ go more than four times a year. All age groups (except respondents aged 60 and up) mostly visit rural tourist destinations once or twice a year).

In Austria, the most respondents aged 40 to $49(33.3 \%)$ go once in two or three years, the most respondents aged 18 to $29(41.3 \%)$ go once or twice a year, the most respondents aged 30 to $39(47.4 \%)$ go three or four times a year, whereas the most 
respondents aged 18 to $29(43.2 \%)$ go more than four times a year. All age groups mostly visit rural tourist destinations once or twice a year).

It can be established that younger groups of respondents (up to 39 years of age) visit rural tourist destinations more often (three, four or more times a year).

Table 3 presents the difference in travel companions according to the respondents' age.

Table 3: Testing the independence of variables: Travel Companions and Respondents' Age

\begin{tabular}{|c|c|c|c|c|c|c|c|c|c|}
\hline \multirow{2}{*}{\multicolumn{4}{|c|}{ Country }} & \multicolumn{5}{|c|}{ Age } & \multirow{3}{*}{$\begin{array}{c}\text { Total } \\
2\end{array}$} \\
\hline & & & & $18-29$ & $30-39$ & $40-49$ & $50-59$ & $\geq 60$ & \\
\hline \multirow{15}{*}{$\frac{\pi}{0}$} & \multirow{15}{*}{$\begin{array}{l}\text { Travel } \\
\text { companions }\end{array}$} & \multirow{3}{*}{ Alone } & Count & 0 & 1 & 0 & 0 & 1 & \\
\hline & & & $\begin{array}{l}\% \\
\text { Companions }\end{array}$ & $0.0 \%$ & $50.0 \%$ & $0.0 \%$ & $0.0 \%$ & $50.0 \%$ & $100.0 \%$ \\
\hline & & & $\%$ Age & $0.0 \%$ & $1.8 \%$ & $0.0 \%$ & $0.0 \%$ & $14.3 \%$ & $1.3 \%$ \\
\hline & & \multirow{3}{*}{ Partner } & Count & 9 & 9 & 4 & 7 & 2 & 31 \\
\hline & & & $\begin{array}{l}\% \\
\text { Companions }\end{array}$ & $29.0 \%$ & $29.0 \%$ & $12.9 \%$ & $22.6 \%$ & $6.5 \%$ & $100.0 \%$ \\
\hline & & & $\%$ Age & $22.5 \%$ & $16.1 \%$ & $13.8 \%$ & $38.9 \%$ & $28.6 \%$ & $20.7 \%$ \\
\hline & & \multirow{3}{*}{ Family } & Count & 22 & 30 & 14 & 7 & 3 & 76 \\
\hline & & & $\begin{array}{l}\text { \% } \\
\text { Companions }\end{array}$ & $28.9 \%$ & $39.5 \%$ & $18.4 \%$ & $9.2 \%$ & $3.9 \%$ & $100.0 \%$ \\
\hline & & & $\%$ Age & $55.0 \%$ & $53.6 \%$ & $48.3 \%$ & $38.9 \%$ & $42.9 \%$ & $50.7 \%$ \\
\hline & & \multirow{3}{*}{ Friends } & Count & 9 & 13 & 11 & 4 & 1 & 38 \\
\hline & & & $\begin{array}{l}\text { \% } \\
\text { Companions }\end{array}$ & $23.7 \%$ & $34.2 \%$ & $28.9 \%$ & $10.5 \%$ & $2.6 \%$ & $100.0 \%$ \\
\hline & & & $\%$ Age & $22.5 \%$ & $23.2 \%$ & $37.9 \%$ & $22.2 \%$ & $14.3 \%$ & $25.3 \%$ \\
\hline & & \multirow{3}{*}{ Other } & Count & 0 & 3 & 0 & 0 & 0 & 3 \\
\hline & & & $\begin{array}{l}\% \\
\text { Companions }\end{array}$ & $0.0 \%$ & $100.0 \%$ & $0.0 \%$ & $0.0 \%$ & $0.0 \%$ & $100.0 \%$ \\
\hline & & & $\%$ Age & $0.0 \%$ & $5.4 \%$ & $0.0 \%$ & $0.0 \%$ & $0.0 \%$ & $2.0 \%$ \\
\hline \multirow{2}{*}{\multicolumn{4}{|c|}{ Country }} & \multicolumn{5}{|c|}{ Age } & \multirow{2}{*}{ Total } \\
\hline & & & & $18-29$ & $30-39$ & $40-49$ & $50-59$ & $\geq 60$ & \\
\hline \multirow{15}{*}{ 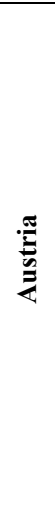 } & \multirow{15}{*}{$\begin{array}{l}\text { Travel } \\
\text { companions }\end{array}$} & \multirow{3}{*}{ Alone } & Count & 0 & 0 & 1 & 0 & 0 & 1 \\
\hline & & & $\%$ Companions & $0.0 \%$ & $0.0 \%$ & $100.0 \%$ & $0.0 \%$ & $0.0 \%$ & $100.0 \%$ \\
\hline & & & $\%$ Age & $0.0 \%$ & $0.0 \%$ & $3.0 \%$ & $0.0 \%$ & $0.0 \%$ & $0.7 \%$ \\
\hline & & \multirow{3}{*}{ Partner } & Count & 9 & 9 & 7 & 3 & 1 & 29 \\
\hline & & & $\%$ Companions & $31.0 \%$ & $31.0 \%$ & $24.1 \%$ & $10.3 \%$ & $3.4 \%$ & $100.0 \%$ \\
\hline & & & $\%$ Age & $17.0 \%$ & $22.0 \%$ & $21.2 \%$ & $18.8 \%$ & $14.3 \%$ & $19.3 \%$ \\
\hline & & \multirow{3}{*}{ Family } & Count & 33 & 24 & 20 & 9 & 4 & 90 \\
\hline & & & $\%$ Companions & $36.7 \%$ & $26.7 \%$ & $22.2 \%$ & $10.0 \%$ & $4.4 \%$ & $100.0 \%$ \\
\hline & & & $\%$ Age & $62.3 \%$ & $58.5 \%$ & $60.6 \%$ & $56.3 \%$ & $57.1 \%$ & $60.0 \%$ \\
\hline & & \multirow{3}{*}{ Friends } & Count & 11 & 8 & 4 & 4 & 2 & 29 \\
\hline & & & $\%$ Companions & $37.9 \%$ & $27.6 \%$ & $13.8 \%$ & $13.8 \%$ & $6.9 \%$ & $100.0 \%$ \\
\hline & & & $\%$ Age & $20.8 \%$ & $19.5 \%$ & $12.1 \%$ & $25.0 \%$ & $28.6 \%$ & $19.3 \%$ \\
\hline & & \multirow{3}{*}{ Other } & Count & 0 & 0 & 1 & 0 & 0 & 1 \\
\hline & & & $\%$ Companions & $0.0 \%$ & $0.0 \%$ & $100.0 \%$ & $0.0 \%$ & $0.0 \%$ & $100.0 \%$ \\
\hline & & & $\%$ Age & $0.0 \%$ & $0.0 \%$ & $3.0 \%$ & $0.0 \%$ & $0.0 \%$ & $0.7 \%$ \\
\hline
\end{tabular}

Source: the authors' calculation 
Based on cross-tabulation of categorical variables Travel Companions and Respondents' Age in Serbia, respondents aged 30 to 39 (50.0\%), and respondents aged 60 and up (50.0\%) travel alone most often, respondents aged 18 to $29(29.0 \%)$ and respondents aged 30 to $39(29.0 \%)$ travel with a partner, respondents aged 30 to $39(39.5 \%)$,) travel with families most often, respondents aged 30 to 39 (34.2\%) travel with friends most often, and 3 respondents aged 30 to 39 travel with other companions (100.0\%).

In Austria, 1 respondent aged 40 to 49 mostly travels alone $(100.0 \%)$, respondents aged 18 to $29(31.0 \%)$ respondents aged 18 to $29(31.0 \%)$ travel with partners most often, respondents aged 18 to $29(36.7 \%)$ travel with families most often, respondents aged 18 to $29(37.9 \%)$ go with friends most often, and 1 respondent aged 40 to $49(100.0 \%)$ mostly travels with other companions.

It can be established that all age groups of respondents in both countries visit rural tourist destinations with families in most cases.

Table 4 presents the differences in information sources used by consumers when planning visits to rural tourist destinations according to the respondents' age.

Table 4: Testing the independence of variables: Information Sources Used to Plan the Trip and Respondents' Age

\begin{tabular}{|c|c|c|c|c|c|c|c|c|c|}
\hline \multirow{2}{*}{\multicolumn{4}{|c|}{ Country }} & \multicolumn{5}{|c|}{ Age } & \multirow{3}{*}{$\begin{array}{c}\text { Total } \\
74 \\
\end{array}$} \\
\hline & & & & \multirow{2}{*}{$\begin{array}{c}18-29 \\
23 \\
\end{array}$} & \multirow{2}{*}{$\begin{array}{c}30-39 \\
27 \\
\end{array}$} & \multirow{2}{*}{$\begin{array}{c}40-49 \\
17 \\
\end{array}$} & \multirow{2}{*}{$\begin{array}{c}50-59 \\
6 \\
\end{array}$} & \multirow{2}{*}{$\begin{array}{c}\geq 60 \\
1\end{array}$} & \\
\hline & \multirow{9}{*}{$\begin{array}{l}\text { Information } \\
\text { sources used to } \\
\text { plan the trip }\end{array}$} & \multirow{3}{*}{ Internet } & Count & & & & & & \\
\hline & & & $\begin{array}{l}\% \text { Inf. } \\
\text { sources }\end{array}$ & $\begin{array}{c}31.1 \\
\%\end{array}$ & $\begin{array}{c}36.5 \\
\%\end{array}$ & $\begin{array}{c}23.0 \\
\%\end{array}$ & $8.1 \%$ & $1.4 \%$ & $\begin{array}{c}100.0 \\
\%\end{array}$ \\
\hline & & & $\%$ Age & $\begin{array}{c}57.5 \\
\%\end{array}$ & $\begin{array}{c}48.2 \\
\%\end{array}$ & $\begin{array}{c}58.6 \\
\%\end{array}$ & $\begin{array}{c}33.3 \\
\%\end{array}$ & $14.3 \%$ & $49.3 \%$ \\
\hline & & \multirow{3}{*}{$\begin{array}{l}\text { Recommendations of friends } \\
\text { and/or relatives }\end{array}$} & Count & 12 & 19 & 11 & 9 & 5 & 56 \\
\hline & & & $\begin{array}{l}\% \text { Inf. } \\
\text { sources } \\
\end{array}$ & $\begin{array}{c}21.4 \\
\% \\
\end{array}$ & $\begin{array}{c}33.9 \\
\% \\
\end{array}$ & $\begin{array}{c}19.6 \\
\% \\
\end{array}$ & $\begin{array}{c}16.1 \\
\% \\
\end{array}$ & $8.9 \%$ & $\begin{array}{c}100.0 \\
\% \\
\end{array}$ \\
\hline & & & $\%$ Age & $\begin{array}{c}30.0 \\
\% \\
\end{array}$ & $\begin{array}{c}33.9 \\
\%\end{array}$ & $\begin{array}{c}37.9 \\
\% \\
\end{array}$ & $\begin{array}{c}50.0 \\
\% \\
\end{array}$ & $71.4 \%$ & $37.3 \%$ \\
\hline & & \multirow{3}{*}{ Own experience } & Count & 5 & 10 & 1 & 3 & 1 & 20 \\
\hline & & & $\begin{array}{l}\% \text { Inf. } \\
\text { sources } \\
\end{array}$ & $\begin{array}{c}25.0 \\
\% \\
\end{array}$ & $\begin{array}{c}50.0 \\
\% \\
\end{array}$ & $5.0 \%$ & $\begin{array}{c}15.0 \\
\% \\
\end{array}$ & $5.0 \%$ & $\begin{array}{c}100.0 \\
\% \\
\end{array}$ \\
\hline & & & $\%$ Age & $\begin{array}{c}12.5 \\
\%\end{array}$ & $\begin{array}{c}17.9 \\
\%\end{array}$ & $3.4 \%$ & $\begin{array}{c}16.7 \\
\%\end{array}$ & $14.3 \%$ & $13.3 \%$ \\
\hline & \multirow{9}{*}{$\begin{array}{l}\text { Information } \\
\text { sources used to } \\
\text { plan the trip }\end{array}$} & \multirow{3}{*}{ Internet } & Count & 31 & 22 & 19 & 5 & 1 & 78 \\
\hline & & & \begin{tabular}{|l|}
$\%$ Inf. \\
sources \\
\end{tabular} & $\begin{array}{c}39.7 \\
\% \\
\end{array}$ & $\begin{array}{c}28.2 \\
\%\end{array}$ & $\begin{array}{c}24.4 \\
\% \\
\end{array}$ & $6.4 \%$ & $1.3 \%$ & $\begin{array}{c}100.0 \\
\%\end{array}$ \\
\hline & & & $\%$ Age & $\begin{array}{c}58.5 \\
\% \\
\end{array}$ & $\begin{array}{c}53.7 \\
\% \\
\end{array}$ & $\begin{array}{c}57.6 \\
\% \\
\end{array}$ & $\begin{array}{c}31.3 \\
\% \\
\end{array}$ & $14.3 \%$ & $52.0 \%$ \\
\hline & & \multirow{3}{*}{$\begin{array}{l}\text { Recommendations of friends } \\
\text { and/or relatives }\end{array}$} & Count & 12 & 16 & 9 & 7 & 5 & 49 \\
\hline & & & \begin{tabular}{|l|}
$\%$ Inf. \\
sources \\
\end{tabular} & $\begin{array}{c}24.5 \\
\% \\
\end{array}$ & $\begin{array}{c}32,7 \\
\% \\
\end{array}$ & $\begin{array}{c}18.4 \\
\% \\
\end{array}$ & $\begin{array}{c}14,3 \\
\% \\
\end{array}$ & $10.2 \%$ & $\begin{array}{c}100.0 \\
\%\end{array}$ \\
\hline & & & $\%$ Age & $\begin{array}{c}22,6 \\
\% \\
\end{array}$ & $\begin{array}{c}39,0 \\
\% \\
\end{array}$ & $\begin{array}{c}27,3 \\
\% \\
\end{array}$ & $\begin{array}{c}43,8 \\
\% \\
\end{array}$ & $71.4 \%$ & $32.7 \%$ \\
\hline & & \multirow{3}{*}{ Own experience } & Count & 6 & 3 & 4 & 4 & 0 & 17 \\
\hline & & & $\begin{array}{l}\% \text { Inf. } \\
\text { sources }\end{array}$ & $\begin{array}{c}35.3 \\
\%\end{array}$ & $\begin{array}{c}17.6 \\
\% \\
\end{array}$ & $\begin{array}{c}23.5 \\
\% \\
\end{array}$ & $\begin{array}{c}23.5 \\
\% \\
\end{array}$ & $0.0 \%$ & $\begin{array}{c}100.0 \\
\% \\
\end{array}$ \\
\hline & & & $\%$ Age & $\begin{array}{c}11.3 \\
\%\end{array}$ & $7.3 \%$ & $\begin{array}{c}12.1 \\
\% \\
\end{array}$ & $\begin{array}{c}25.0 \\
\% \\
\end{array}$ & $0.0 \%$ & $11.3 \%$ \\
\hline
\end{tabular}




\begin{tabular}{|c|c|c|c|c|c|c|c|c|c|}
\hline & & \multirow{3}{*}{$\begin{array}{l}\text { Newspapers/magazines/catal } \\
\text { ogues }\end{array}$} & Count & 0 & 0 & 0 & 0 & 1 & 1 \\
\hline & & & $\begin{array}{l}\% \text { Inf. } \\
\text { sources }\end{array}$ & $0.0 \%$ & $0.0 \%$ & $0.0 \%$ & $0.0 \%$ & $\begin{array}{c}100.0 \\
\%\end{array}$ & $\begin{array}{c}100.0 \\
\%\end{array}$ \\
\hline & & & $\%$ Age & $0.0 \%$ & $0.0 \%$ & $0.0 \%$ & $0.0 \%$ & $14.3 \%$ & $0.7 \%$ \\
\hline & & \multirow{3}{*}{ Travel agencies } & Count & 4 & 0 & 1 & 0 & 0 & 5 \\
\hline & & & $\begin{array}{l}\% \text { Inf. } \\
\text { sources }\end{array}$ & $\begin{array}{c}80.0 \\
\%\end{array}$ & $0.0 \%$ & $\begin{array}{c}20.0 \\
\%\end{array}$ & $0.0 \%$ & $0.0 \%$ & $\begin{array}{c}100.0 \\
\%\end{array}$ \\
\hline & & & $\%$ Age & $7.5 \%$ & $0.0 \%$ & $3.0 \%$ & $0.0 \%$ & $0.0 \%$ & $3.3 \%$ \\
\hline
\end{tabular}

Source: the authors' calculation

Based on cross-tabulation of categorical variables Sources Used by Consumers when Planning Visits to Rural Tourist Destinations and Respondents' Age in Serbia, the Internet is used most often by respondents aged 30 to $39(36.5 \%)$ friends' and relatives' recommendations are used most often by respondents aged 30 to 39 (33.9\%), whereas planning travel based on their own experience is practiced most often by respondents aged 30 to $39(50.0 \%)$.

In Austria, the Internet is used most often by respondents aged 18 to $29(39.7 \%)$, friends' and/or relatives' recommendations are used most often by respondents aged 30 to $39(32.7 \%)$, planning travel based on their own experience is practiced most often by respondents aged 18 to $29(35.3 \%)$, newspapers/magazines/catalogues are used by 1 respondent aged 60 and up (100\%), and travel agencies are mostly consulted by respondents aged 18 to 29 .

It can be established that respondents aged up to 49 use the Internet for planning visits to rural tourist destinations, most often, whereas respondents aged 50 and up mostly listen to friends' and relatives' recommendations when planning visits to rural tourist destinations.

Based on the results obtained by cross-tabulation, it can be concluded that respondents aged up to 39 visit rural tourist destinations more often, that all groups of respondents visit rural tourist destinations with families most often; furthermore, that respondents aged up to 49 use the Internet most often to plan visits to plan visits to rural tourist destinations, whereas the elderly mostly listen to friends' and relatives' recommendations when planning visits to rural tourist destinations.

Table 5: Testing the independence of variables: Frequency of Engaging in Rural Tourism and Respondent's Education Level

\begin{tabular}{|c|c|c|c|c|c|c|c|c|c|}
\hline \multirow{2}{*}{\multicolumn{4}{|c|}{ Country }} & \multicolumn{5}{|c|}{ Education level } & \multirow{3}{*}{$\begin{array}{c}\text { Total } \\
35 \\
\end{array}$} \\
\hline & & & & \multirow{2}{*}{$\begin{array}{c}\begin{array}{c}\text { Primary } \\
\text { school }\end{array} \\
1 \\
\end{array}$} & \multirow{2}{*}{$\begin{array}{c}\begin{array}{c}\text { Secondary } \\
\text { school }\end{array} \\
8 \\
\end{array}$} & \multirow{2}{*}{$\begin{array}{c}\text { Bachelor } \\
12 \\
\end{array}$} & \multirow{2}{*}{$\begin{array}{c}\text { Master } \\
9\end{array}$} & \multirow{2}{*}{\begin{tabular}{|c|} 
Doctoral \\
5 \\
\end{tabular}} & \\
\hline \multirow{6}{*}{ 愛 } & \multirow{6}{*}{$\begin{array}{l}\text { Frequency } \\
\text { of engaging } \\
\text { in rural } \\
\text { tourism }\end{array}$} & \multirow{3}{*}{$\begin{array}{l}\text { Once every } \\
\text { two or three } \\
\text { years }\end{array}$} & Count & & & & & & \\
\hline & & & $\begin{array}{l}\% \\
\text { Frequency }\end{array}$ & $2.9 \%$ & $22.9 \%$ & $34.3 \%$ & $25.7 \%$ & $14.3 \%$ & $100.0 \%$ \\
\hline & & & $\%$ Age & $100.0 \%$ & $36.4 \%$ & $17.6 \%$ & $24.3 \%$ & $22.7 \%$ & $23.3 \%$ \\
\hline & & \multirow{3}{*}{$\begin{array}{l}\text { Once or } \\
\text { twice a year }\end{array}$} & Count & 0 & 4 & 30 & 11 & 9 & 54 \\
\hline & & & \begin{tabular}{|l|}
$\%$ \\
Frequency \\
\end{tabular} & $0.0 \%$ & $7.4 \%$ & $55.6 \%$ & $20.4 \%$ & $16.7 \%$ & $100.0 \%$ \\
\hline & & & $\%$ Age & $0.0 \%$ & $18.2 \%$ & $44.1 \%$ & $29.7 \%$ & $40.9 \%$ & $36.0 \%$ \\
\hline
\end{tabular}




\begin{tabular}{|c|c|c|c|c|c|c|c|c|c|}
\hline & & & Count & 0 & 6 & 13 & 10 & 5 & 34 \\
\hline & & $\begin{array}{l}\text { Three or four } \\
\text { times a year }\end{array}$ & \begin{tabular}{|l|}
$\%$ \\
Frequency
\end{tabular} & $0.0 \%$ & $17.6 \%$ & $38.2 \%$ & $29.4 \%$ & $14.7 \%$ & $100.0 \%$ \\
\hline & & & \% Age & $0.0 \%$ & $27.3 \%$ & $19.1 \%$ & $27.0 \%$ & $22.7 \%$ & $22.7 \%$ \\
\hline & & & Count & 0 & 4 & 13 & 7 & 3 & 27 \\
\hline & & $\begin{array}{l}\text { More than } \\
\text { four times a }\end{array}$ & \begin{tabular}{|l|}
$\%$ \\
Frequency \\
\end{tabular} & $0.0 \%$ & $14.8 \%$ & $48.1 \%$ & $25.9 \%$ & $11.1 \%$ & $100.0 \%$ \\
\hline & & & $\%$ Age & $0.0 \%$ & $18.2 \%$ & $19.1 \%$ & $18.9 \%$ & $13.6 \%$ & $18.0 \%$ \\
\hline \multirow{12}{*}{ 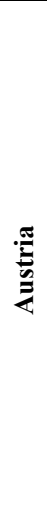 } & \multirow{12}{*}{$\begin{array}{l}\text { Frequency } \\
\text { of engaging } \\
\text { in rural } \\
\text { tourism }\end{array}$} & \multirow{3}{*}{$\begin{array}{l}\text { Once every } \\
\text { two or three } \\
\text { years }\end{array}$} & Count & 1 & 7 & 19 & 7 & 8 & 42 \\
\hline & & & \begin{tabular}{|l|}
$\%$ \\
Frequency \\
\end{tabular} & $2.4 \%$ & $16.7 \%$ & $45.2 \%$ & $16.7 \%$ & $19.0 \%$ & $100.0 \%$ \\
\hline & & & $\%$ Age & $100.0 \%$ & $28.0 \%$ & $25.7 \%$ & $25.9 \%$ & $34.8 \%$ & $28.0 \%$ \\
\hline & & \multirow{3}{*}{$\begin{array}{l}\text { Once or } \\
\text { twice a year }\end{array}$} & Count & 0 & 6 & 26 & 11 & 5 & 48 \\
\hline & & & \begin{tabular}{|l|}
$\%$ \\
Frequency
\end{tabular} & $0.0 \%$ & $12.5 \%$ & $54.2 \%$ & $22.9 \%$ & $10.4 \%$ & $100.0 \%$ \\
\hline & & & $\%$ Age & $0.0 \%$ & $24.0 \%$ & $35.1 \%$ & $40.7 \%$ & $21.7 \%$ & $32.0 \%$ \\
\hline & & \multirow{3}{*}{$\begin{array}{l}\text { Three or four } \\
\text { times a year }\end{array}$} & Count & 0 & 1 & 5 & 5 & 5 & 16 \\
\hline & & & \begin{tabular}{|l|}
$\%$ \\
Frequency \\
\end{tabular} & $0.0 \%$ & $6.3 \%$ & $31.3 \%$ & $31.3 \%$ & $31.3 \%$ & $100.0 \%$ \\
\hline & & & $\%$ Age & $0.0 \%$ & $4.0 \%$ & $6.8 \%$ & $18.5 \%$ & $21.7 \%$ & $10.7 \%$ \\
\hline & & \multirow{3}{*}{$\begin{array}{l}\text { More than } \\
\text { four times a } \\
\text { year }\end{array}$} & Count & 0 & 11 & 24 & 4 & 5 & 44 \\
\hline & & & $\begin{array}{l}\% \\
\text { Frequency }\end{array}$ & $0.0 \%$ & $25.0 \%$ & $54.5 \%$ & $9.1 \%$ & $11.4 \%$ & $100.0 \%$ \\
\hline & & & \% Age & $0.0 \%$ & $44.0 \%$ & $32.4 \%$ & $14.8 \%$ & $21.7 \%$ & $29.3 \%$ \\
\hline
\end{tabular}

Source: the authors' calculation

Based on cross tabulation of categorical variables Frequency of engaging in rural tourism (i.e. visits to rural tourist destinations) and Respondents' education levels in Serbia, respondents with Bachelor's degrees $(34.3 \%)$ go once in two or three years most often, respondents with Bachelor's degrees (55.6\%) go once or twice a year most often, respondents with Bachelor's degrees $(38.2 \%)$ go three or four times a year most often, respondents with Bachelor's degrees (48.1\%) go more than four times a year. Groups of respondents with elementary or secondary education visit rural tourist destinations in most cases once in two or three years, while respondents with Bachelor's or higher degrees visit rural tourist destinations in most cases once or twice a year.

In Austria, respondents with Bachelor's degrees (45.2\%) go once in two or three years most often, respondents with Bachelor's degrees $(54.2 \%)$ go once or twice a year most often, respondents with Bachelor's (31.3\%), Master's (31.3\%) and PhD degrees $(31.3 \%)$ go three or four times a year most often, and respondents with Bachelor's degrees go more than four times a year. Groups of respondents with elementary education and $\mathrm{PhD}$ degrees visit rural tourist destinations in most cases once in two or three years whereas respondents with Bachelor's or Master's degrees visit rural tourist destinations in most cases three or four times a year.

It can be established that respondents with Bachelor's degrees visit rural tourist destinations more often (three, four or more than four times a year).

Table 6 presents differences in travel companions according to the respondents' education levels. 
Table 6: Testing the independence of variables: Travel Companions and Respondents' Education Level

\begin{tabular}{|c|c|c|c|c|c|c|c|c|c|}
\hline & & & & $\begin{array}{c}\text { Primary } \\
\text { school }\end{array}$ & $\begin{array}{c}\text { Secondary } \\
\text { school }\end{array}$ & Bachelor & Master & Doctoral & \\
\hline \multirow{15}{*}{ } & \multirow{15}{*}{$\begin{array}{l}\text { Travel } \\
\text { companions }\end{array}$} & \multirow{3}{*}{ Alone } & Count & 0 & 0 & 2 & 0 & 0 & 2 \\
\hline & & & \begin{tabular}{|l|}
$\%$ \\
Companions \\
\end{tabular} & $0.0 \%$ & $0.0 \%$ & $100.0 \%$ & $0.0 \%$ & $0.0 \%$ & $100.0 \%$ \\
\hline & & & $\%$ Age & $0.0 \%$ & $0.0 \%$ & $2.9 \%$ & $0.0 \%$ & $0.0 \%$ & $1.3 \%$ \\
\hline & & \multirow{3}{*}{ Partner } & Count & 0 & 5 & 14 & 7 & 5 & 31 \\
\hline & & & $\begin{array}{l}\% \\
\text { Companions }\end{array}$ & $0.0 \%$ & $16.1 \%$ & $45.2 \%$ & $22.6 \%$ & $16.1 \%$ & $100.0 \%$ \\
\hline & & & $\%$ Age & $0.0 \%$ & $22.7 \%$ & $20.6 \%$ & $18.9 \%$ & $22.7 \%$ & $20.7 \%$ \\
\hline & & \multirow{3}{*}{ Family } & Count & 1 & 12 & 31 & 19 & 13 & 76 \\
\hline & & & \begin{tabular}{|l}
$\%$ \\
Companions \\
\end{tabular} & $1.3 \%$ & $15.8 \%$ & $40.8 \%$ & $25.0 \%$ & $17.1 \%$ & $100.0 \%$ \\
\hline & & & $\%$ Age & $100.0 \%$ & $54.5 \%$ & $45.6 \%$ & $51.4 \%$ & $59.1 \%$ & $50.7 \%$ \\
\hline & & \multirow{3}{*}{ Friends } & Count & 0 & 5 & 19 & 10 & 4 & 38 \\
\hline & & & $\begin{array}{l}\% \\
\text { Companions } \\
\end{array}$ & $0.0 \%$ & $13.2 \%$ & $50.0 \%$ & $26.3 \%$ & $10.5 \%$ & $100.0 \%$ \\
\hline & & & $\%$ Age & $0.0 \%$ & $22.7 \%$ & $27.9 \%$ & $27.0 \%$ & $18.2 \%$ & $25.3 \%$ \\
\hline & & \multirow{3}{*}{ Other } & Count & 0 & 0 & 2 & 1 & 0 & 3 \\
\hline & & & \begin{tabular}{|l|}
$\%$ \\
Companions \\
\end{tabular} & $0.0 \%$ & $0.0 \%$ & $66.7 \%$ & $33.3 \%$ & $0.0 \%$ & $100.0 \%$ \\
\hline & & & $\%$ Age & $0.0 \%$ & $0.0 \%$ & $2.9 \%$ & $2.7 \%$ & $0.0 \%$ & $2.0 \%$ \\
\hline \multirow{15}{*}{ 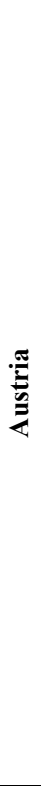 } & \multirow{15}{*}{$\begin{array}{l}\text { Travel } \\
\text { companions }\end{array}$} & \multirow{3}{*}{ Alone } & Count & 0 & 0 & 1 & 0 & 0 & 1 \\
\hline & & & $\begin{array}{l}\% \\
\text { Companions } \\
\end{array}$ & $0.0 \%$ & $0.0 \%$ & $100.0 \%$ & $0.0 \%$ & $0.0 \%$ & $100.0 \%$ \\
\hline & & & $\%$ Age & $0.0 \%$ & $0.0 \%$ & $1.4 \%$ & $0.0 \%$ & $0.0 \%$ & $0.7 \%$ \\
\hline & & \multirow{3}{*}{ Partner } & Count & 0 & 5 & 10 & 10 & 4 & 29 \\
\hline & & & \begin{tabular}{|l|}
$\%$ \\
Companions \\
\end{tabular} & $0.0 \%$ & $17.2 \%$ & $34.5 \%$ & $34.5 \%$ & $13.8 \%$ & $100.0 \%$ \\
\hline & & & $\%$ Age & $0.0 \%$ & $20.0 \%$ & $13.5 \%$ & $37.0 \%$ & $17.4 \%$ & $19.3 \%$ \\
\hline & & \multirow{3}{*}{ Family } & Count & 1 & 15 & 44 & 15 & 15 & 90 \\
\hline & & & \begin{tabular}{|l|}
$\%$ \\
Companions \\
\end{tabular} & $1.1 \%$ & $16.7 \%$ & $48.9 \%$ & $16.7 \%$ & $16.7 \%$ & $100.0 \%$ \\
\hline & & & $\%$ Age & $100.0 \%$ & $60.0 \%$ & $59.5 \%$ & $55.6 \%$ & $65.2 \%$ & $60.0 \%$ \\
\hline & & \multirow{3}{*}{ Friends } & Count & 0 & 5 & 18 & 2 & 4 & 29 \\
\hline & & & \begin{tabular}{|l|}
$\%$ \\
Companions \\
\end{tabular} & $0.0 \%$ & $17.2 \%$ & $62.1 \%$ & $6.9 \%$ & $13.8 \%$ & $100.0 \%$ \\
\hline & & & $\%$ Age & $0.0 \%$ & $20.0 \%$ & $24.3 \%$ & $7.4 \%$ & $17.4 \%$ & $19 ., 3 \%$ \\
\hline & & \multirow{3}{*}{ Other } & Count & 0 & 0 & 1 & 0 & 0 & 1 \\
\hline & & & $\begin{array}{l}\% \\
\text { Companions }\end{array}$ & $0.0 \%$ & $0,0 \%$ & $100,0 \%$ & $0.0 \%$ & $0.0 \%$ & $100.0 \%$ \\
\hline & & & $\%$ Age & $0,0 \%$ & $0,0 \%$ & $1.4 \%$ & $0,0 \%$ & $0,0 \%$ & $0,7 \%$ \\
\hline
\end{tabular}

Source: Author's calculation 
Based on cross-tabulation of categorical variables Travel Companions and Respondents' Education Levels, 2 respondents (100.0\%) with Bachelor's degrees travel alone most often, respondents with Bachelor's degrees $(45.2 \%)$ travel with partners most often, respondents with Bachelor's degrees travel with families $(40.8 \%)$ most often, respondents with Bachelor's degrees travel with friends $(50.0 \%)$ most often, and 2 respondents with Bachelor's degrees (100.0\%) travel with other companions.

In Austria, 1 respondent with Bachelor's degrees travels alone most often, respondents with Bachelor's and Master's degrees (34,5\%) travel with partners most often, respondents with Bachelor's degrees travel with families $(48,9 \%)$ most often, respondents with Bachelor's degrees travel with friends $(62,1 \%)$ most often, and 1 respondent with Bachelor's degrees (100.0\%) travels with other companions.

It can be established that respondents of all education levels in both states visit rural destinations with families most often.

Table 7 presents differences in information sources used by consumers when planning visits to rural tourist destinations according to respondents' education levels.

Table 7: Testing the independence of variables: Information Sources Used to Plan the Trip and Respondents' Education Level

\begin{tabular}{|c|c|c|c|c|c|c|c|c|c|}
\hline \multirow{2}{*}{\multicolumn{4}{|c|}{ Country }} & \multicolumn{5}{|c|}{ Education level } & \multirow{3}{*}{$\begin{array}{c}\text { Total } \\
74\end{array}$} \\
\hline & & & & \multirow{2}{*}{$\begin{array}{c}\begin{array}{c}\text { Primary } \\
\text { school }\end{array} \\
0\end{array}$} & \multirow{2}{*}{$\begin{array}{c}\begin{array}{c}\text { Secondary } \\
\text { school }\end{array} \\
10 \\
\end{array}$} & \multirow{2}{*}{$\begin{array}{c}\text { Bachelo } \\
\text { r }\end{array}$} & \multirow{2}{*}{$\begin{array}{c}\text { Maste } \\
\text { r } \\
20\end{array}$} & \multirow{2}{*}{$\begin{array}{c}\text { Doctor } \\
\text { al } \\
7\end{array}$} & \\
\hline \multirow{9}{*}{ 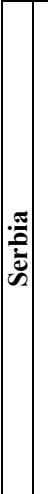 } & \multirow{9}{*}{$\begin{array}{l}\text { Informati } \\
\text { on } \\
\text { sources } \\
\text { used to } \\
\text { plan the } \\
\text { trip }\end{array}$} & \multirow{3}{*}{ Internet } & Count & & & & & & \\
\hline & & & $\begin{array}{l}\% \text { Inf. } \\
\text { sources }\end{array}$ & $0.0 \%$ & $13.5 \%$ & $50.0 \%$ & $\begin{array}{c}27.0 \\
\%\end{array}$ & $9.5 \%$ & $\begin{array}{c}100.0 \\
\%\end{array}$ \\
\hline & & & $\%$ Age & $0.0 \%$ & $45.5 \%$ & $54.4 \%$ & $\begin{array}{c}54.1 \\
\% \\
\end{array}$ & $31.8 \%$ & $49.3 \%$ \\
\hline & & \multirow{3}{*}{$\begin{array}{l}\text { Recommendations of } \\
\text { friends and/or relatives }\end{array}$} & Count & 0 & 9 & 25 & 10 & 12 & 56 \\
\hline & & & $\begin{array}{l}\% \text { Inf. } \\
\text { sources }\end{array}$ & $0.0 \%$ & $16.1 \%$ & $44.6 \%$ & $\begin{array}{c}17.9 \\
\%\end{array}$ & $21.4 \%$ & $\begin{array}{c}100.0 \\
\%\end{array}$ \\
\hline & & & $\%$ Age & $0.0 \%$ & $40.9 \%$ & $36.8 \%$ & $\begin{array}{c}27.0 \\
\%\end{array}$ & $54.5 \%$ & $37.3 \%$ \\
\hline & & \multirow{3}{*}{ Own experience } & Count & 1 & 3 & 6 & 7 & 3 & 20 \\
\hline & & & $\begin{array}{l}\% \text { Inf. } \\
\text { sources }\end{array}$ & $5.0 \%$ & $15.0 \%$ & $30.0 \%$ & $\begin{array}{c}35.0 \\
\%\end{array}$ & $15.0 \%$ & $\begin{array}{c}100.0 \\
\%\end{array}$ \\
\hline & & & $\%$ Age & $100.0 \%$ & $13.6 \%$ & $8.8 \%$ & $\begin{array}{c}18.9 \\
\%\end{array}$ & $13.6 \%$ & $13.3 \%$ \\
\hline \multirow{7}{*}{ 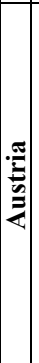 } & \multirow{7}{*}{$\begin{array}{l}\text { Informati } \\
\text { on } \\
\text { sources } \\
\text { used to } \\
\text { plan the } \\
\text { trip }\end{array}$} & \multirow{3}{*}{ Internet } & Count & 0 & 12 & 41 & 18 & 7 & 78 \\
\hline & & & $\begin{array}{l}\% \text { Inf. } \\
\text { sources }\end{array}$ & $0.0 \%$ & $15.4 \%$ & $52.6 \%$ & $\begin{array}{c}23.1 \\
\% \\
\end{array}$ & $9.0 \%$ & $\begin{array}{c}100.0 \\
\%\end{array}$ \\
\hline & & & $\%$ Age & $0.0 \%$ & $48.0 \%$ & $55.4 \%$ & $\begin{array}{c}66.7 \\
\%\end{array}$ & $30.4 \%$ & $52.0 \%$ \\
\hline & & \multirow{3}{*}{$\begin{array}{l}\text { Recommendations of } \\
\text { friends and/or relatives }\end{array}$} & Count & 1 & 10 & 19 & 7 & 12 & 49 \\
\hline & & & $\begin{array}{l}\% \text { Inf. } \\
\text { sources }\end{array}$ & $2.0 \%$ & $20.4 \%$ & $38.8 \%$ & $\begin{array}{c}14.3 \\
\% \\
\end{array}$ & $24.5 \%$ & $\begin{array}{c}100.0 \\
\%\end{array}$ \\
\hline & & & $\%$ Age & $100.0 \%$ & $40.0 \%$ & $25.7 \%$ & $\begin{array}{c}25.9 \\
\%\end{array}$ & $52.2 \%$ & $32.7 \%$ \\
\hline & & Own experience & Count & 0 & 2 & 10 & 2 & 3 & 17 \\
\hline
\end{tabular}




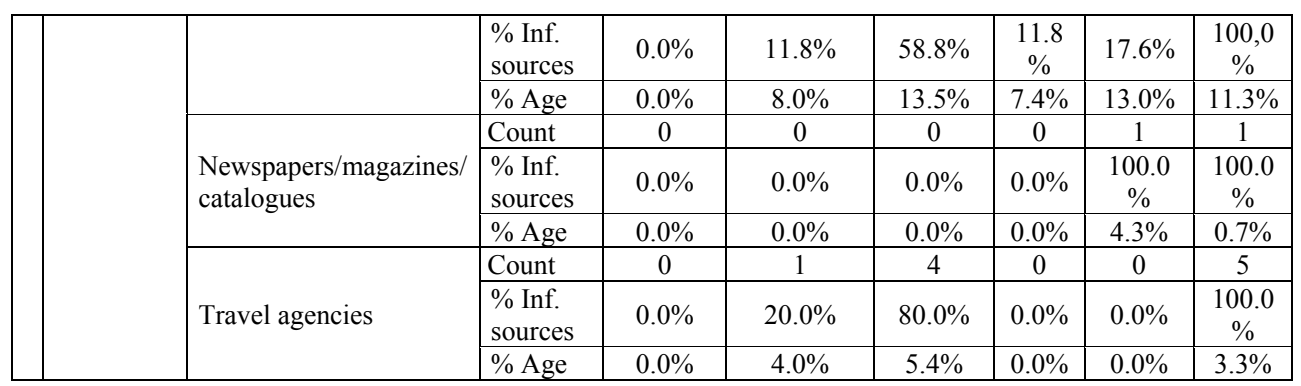

Source: Author's calculation

Based on cross-tabulation of categorical variables Information Sources Used to Plan the Trip and Respondents' Education Level the Internet is used most often by respondents with Bachelors' degrees (50.0\%) friends' and/or relatives' recommendations are used most often by respondents with Bachelors' degrees (44.6\%), and planning travel according to their own experience is used most often by respondents with Master's degrees $(35.0 \%)$.)

In Austria, the Internet is used most often by respondents with Bachelors' degrees (38.8\%) planning travel based on their own experience is practiced most often by respondents with Bachelor's degrees (58.8\%), newspapers/magazines/catalogues are used by one respondent with a $\mathrm{PhD}$ degree $(100.0 \%)$, and travel agencies are consulted most often by respondents with Bachelor's degrees (800\%).

It can be established that, in both countries, respondents with completed secondary school, Bachelor's and Master's degree plan visits to rural tourist destinations most often based on their own experience and friends' and/or relatives' recommendations, whereas respondents with $\mathrm{PhD}$ degrees plan visits to rural tourist destinations based on friends' and/or relatives' recommendation most often.

It can be concluded based on the results obtained by cross-tabulation that respondents with Bachelors' degrees visit rural tourist destinations more often, that all groups of respondents visit rural tourist destinations with families, that respondents with completed secondary schools, Bachelor's and Master's degrees use the Internet most often to plan visits to rural tourist destination, those with elementary education, in most cases, plan visits based on their own experience and friends' and/or relatives' recommendations, whereas respondents with $\mathrm{PhD}$ degrees plan their visits to rural tourist destinations most often based on and friends' and relatives recommendations. Bearing in mind all of the above, it can be concluded that the set hypothesis $\mathrm{H} 2$ is adopted, i.e. that respondents with Bachelor's degrees visit rural tourist destinations more often, and together with respondents with completed secondary school and Master's degrees use the Internet for planning visits to rural tourist destinations, while all education levels of respondents visit rural tourist destinations with families most often. 


\section{Conclusion}

In both countries covered by this research, rural tourism can play a key role in terms of advancing local economy, thereby creating new job opportunities which will generate additional incomes for rural households and, at the same time, it can help maintain or repopulate rural destinations. Rural tourism enriches the tourist offer and provides new quality to the development of tourism in both countries.

The geographical diversity of both countries provides the basis for further planning and actions in the development of rural tourism and its special forms such as homestead and wine tourism. In Serbia, especially in Vojvodina, tourism is one of the key pillars of economic development (Đorđević et al., 2019). Multiculturalism is what makes this region specific and that is why it should invest in development of destinations with tourist and hospitality, foster its authenticity, and exploit the preserved potential and unspoilt nature. (Tomić \& Stoiljković, 2013). In Austria, rural tourism has a long history, and, as such, features as the instrument for restructuring the agricultural sector. In the future, it should have the task to strategically use the potential of touristically attractive rural destinations.

Identifying profiles of potential consumers, i.e. tourists plays a key role in the formation of tourist offer. This paper identifies the profile of tourists visiting rural tourist destinations - homesteads and wineries - in Serbia and Austria. The research's limitation is a small sample, as well as the fact that it covered only a part of Serbia - Vojvodina - and a part of Austria - the area surrounding Vienna. Another limitation is that it only covers two forms of rural tourism - farms and wineries. Thus, in future research, the sample should be expanded, territory researched should be extended, and some more forms of rural tourism should be included into the scope of research.

\section{References}

Alcaniz, E.B., Garcia, I.S., \& Blas, S.S. (2009). The functional-psychological continuum in the cognitive image of a destination: A confirmatory analysis. Tourism Management, 30(5), 715-723. Doi: https://doi.org/10.1016/j.tourman.2008.10.020

Breiling, M. (2005). Rural Tourism: Experiences from Austria, Opportunities for Japan. In: Japanese ž Rural Planning Society, Kinki Meeting, 1-15.

Collins, D., \& Tisdell, C. (2002). Gender and differences in travel life cycles. Journal of Travel Research, 41(2), 133-143. Doi: https://doi.org/10.1177\%2F004728702237413

Cooper, D.R., \& Schindler, P.S. (2006). Business Research Methods. USA: McGraw-Hill.

Đenadić, M., Muhi, B., \& Jovanović, D.V. (2016). Rural tourism - Serbia's missed chance. Economics of Agriculture, 2, 515-529. Doi: https://doi.org/10.5937/ekoPolj1602515D

Đorđević, D., Šušić, V., \& Janjić, I. (2019). Perspectives of development of rural tourism of the Republic of Serbia. Economic Themes, 57(2), 219-232. Doi: https://doi.org/10.2478/ethemes-2019-0013 
Erdeji, I., Gagić, S., Jovičić, A., \& Medić, S. (2013). Development of Rural Tourism in Serbia. Journal of Settlements and Spatial Planning, Special Issue, 2, 309-315.

Foot, D. (2004). Leisure futures: a change in demography? In: Weiermair, K., Mathies, C. (Eds) The Tourism and Leisure Industry: Shaping the Future, (pp. 21-33). The Haworth Hospitality Press.

Grubor, A., Leković, K., \& Tomić, S. (2019). Rural tourism marketing of the Danube region. Ekonomika, 65(4), 1-10. Doi: https://doi.org/10.5937/ekonomika1904001G

Hall, M.C., Sharples, L., Cambourne, B., \& Macionas, N. (2002). Wine Tourism around the World-Developments, management and markets. London, New York: Taylor \& Francis.

Kara, N.S., \& Mkwizu, K.H. (2020). Demographic factors and travel motivation among leisure tourists in Tanzania. International Hospitality Review, 34(1), 81-103. Doi: https://doi.org/10.1108/IHR-01-2020-0002

Koteski, C., Majhošev, D., \& Jakovljev, Z. (2017). Possibilities for the development of rural tourism in the Republic of Macedonia. Journal of Process Management - New Technologies, 5(2), 18-24. Doi: https://doi.org/10.5937/jouproman5-13488

Ma, A., Chow, A., Cheung, L., Lee, K., \& Liu, S. (2018). Impacts of tourists' sociodemographic characteristics on the travel motivation and satisfaction: the case of protected areas in South China. Sustainability, 10(10), 1-21. Doi: https://doi.org/10.3390/su10103388

Medojević, J., Milosavljević, S., \& Punišić, M. (2011). Paradigms of rural tourism in Serbia in the function of village revitalisation. Human Geographies - Journal of Studies and Research in Human Geography, 5(2), 93-102. Doi: https://doi.org/10.5719/hgeo.2011.52.93

Polo Pena, A.I., Jamilena, D.M.F., \& Molina, M.A.R. (2012). Validation of cognitive image dimensions for rural tourist destinations: A contribution to the management of rural tourist destinations. Journal of Vacation Marketing, 18(4), 261-273. Doi: https://doi.org/10.1177\%2F1356766712449351

Quendler, E. (2019). The position of the farm holiday in Austrian tourism. Open Agriculture, 4(1), 697-711. Doi: https://doi.org/10.1515/opag-2019-0069

Radović, G., \& Vasiljević, Z. (2018). Current forms of rural tourism in Serbia and modalities of financing. In:Thematic Proceedings of 3rd International Scientific Conference, Vrnjačka Banja, pp. 521-537.

Stepanov, S., Jovičić, M., \& Jovičić, M. (2018). Strategic positioning sustainable development of rural tourism in Serbia. In: Congress Proceedings of 4th International Rural Tourism Congress, pp. 240-252.

Tomić, S., \& Stoiljković, A. (2013). Tourist movement in Serbia. Anali Ekonomskog fakulteta u Subotici, 49(30), 81-96. 
Vasiljević, Z., \& Vujović, D. (2012). Vinski turizam i njegov značaj za razvoj lokalne zajednice. In: Tourism: Challenges and Opportunities. Ekonomski institut, Beograd, pp. 385-390.

Vujko, A., Petrović, M., Demirović D., \& Racković, I. (2017). Vrednovanje razvoja turizma na salašima Bačke. Agroekonomika, 46(75), 53-62. 